\title{
Chapter
}

\section{CALLIGRAPHY PAPER-BASED ANALYTICAL DEVICES}

\section{Chen-Meng Kuan, ${ }^{1}$ Kuan-Hung Chen, ${ }^{2}$ Min-Wei Hung, ${ }^{3}$ and Chao-Min Cheng4 ${ }^{*}$}

${ }^{1}$ Institute of Nanoengineering and Microsystems, National Tsing Hua University, Hsinchu 30013, Taiwan

2 Institute of Nanoengineering and Microsystems, National Tsing Hua University, Hsinchu 30013, Taiwan

${ }^{3}$ Instrument Technology Research Center, National Applied Research Laboratories, Hsinchu 30076, Taiwan

${ }^{4}$ Institute of Biomedical Engineering, National Tsing Hua University, Hsinchu 30013, Taiwan 


\section{Contents}

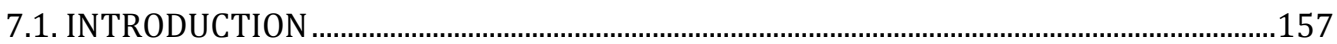

7.2. CALLIGRAPHY PAPER-BASED ANALYTICAL DEVICES ….................................................159

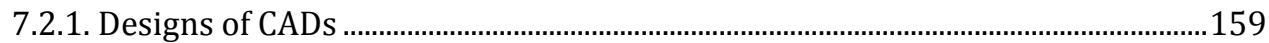

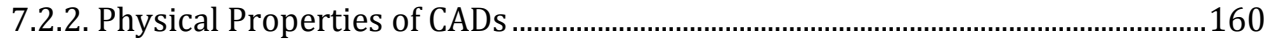

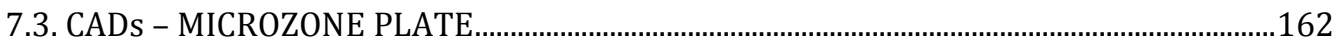

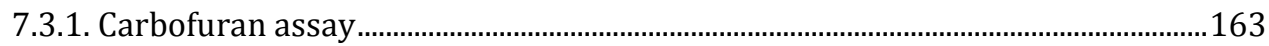

7.4. CADs - HYDROPHOBIC REACTION PLATE ...........................................................................164

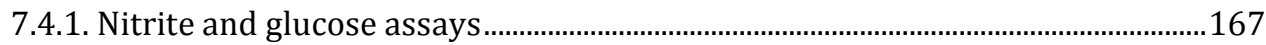

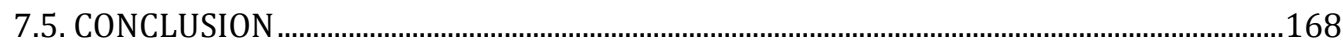

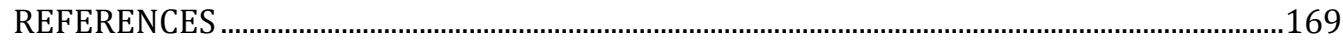




\subsection{INTRODUCTION}

Paper has been broadly used in point-of-care testing (POCT) and chemical analyses for biological and environmental applications [1-4]. Compared to conventional analytical methods commonly used in medical centers, paper is recognized as a promising platform substrate for the development of alternative, inexpensive, and easy-to-use analytical devices [paper-based analytical devices (PADs)] for on-site detections in resource-constrained settings [5-9]. Such devices are conveniently carried, reagent / sample saving (each operation only requires a few liquid microliters), robust, easily disposed of via incineration, and are operable without additional instrumentation. Among the currently developed PADs, their core design includes a reaction area and fluidic channels created via the hydrophilic nature of the paper used [10]. Most existing PADs are built on one of two colorimetric detection approaches, a metabolic assay [e.g., the aspartate aminotransferase (AST) assay, glucose assay, protein assay, etc.] or an enzyme-linked immunosorbent assay (ELISA) [i.e., using antibodies to indicate specific target antigens, such as vascular endothelial growth factor (VEGF), hCG, NC16A, etc.] [2,5,11-13]. Both PAD approaches offer promising strategies to ameliorate global health issues. In addition to analytical use, paper has also become an attractive material for the construction of high-tech devices [e.g., electronic displays, consumer electronics, energy storage devices, microelectromechanical systems (MEMS) sensors, etc.], given its advantageous properties of affordability, flexibility, disposability, porosity, and adaptability to large-scale manufacturing [14-17]. For these applications, the surface chemistry of paper, or at least a portion of it, is likely to be transformed from hydrophilic to hydrophobic to eliminate wetting behaviors native to unaltered paper that might degenerate the mechanical and electrical performances of these devices. However, the methods of transformation (e.g., vapor-phase deposition and micro-transfer printing) are complicated and expensive for small- / medium-sized research laboratories, which are often lacking in experimental resources [16,17].

In this study, we explored the characteristics of two types of papers commonly used in calligraphy-Chinese calligraphy paper and rice paper-to make calligraphy paper-based analytical devices (CADs). These are alternatives to the filter paper commonly employed to make PADs. Chinese calligraphy paper and rice paper are ubiquitous materials in the Far East and are widely used for calligraphy art. With regard to fabrication simplicity, we successfully demonstrated that a commercial wax printer could be used to develop two different CADs: i) a microzone plate, which consists of hydrophilic reaction zones (unaltered papers) and hydrophobic boundaries (wax-covered area); and, ii) a hydrophobic reaction plate, which provides a completely hydrophobic platform for analytical applications. The commercial wax printer provided several advantages: i) the amount of printing wax used during each 
printing cycle was optimized via the manufacturing company; ii) the manipulations for pattern design and printing are user-friendly; and, iii) the choices of wax color can be varied with research demands.

We discovered that CADs demonstrated significantly enhanced assay signal for our carbofuran assay (colorimetric assay) compared to PADs made up of filter paper. Moreover, we took advantage of different wax colors to create reaction plate background designs that lowered the limits of detection (LODs) for colorimetric assays (i.e., nitrite and glucose assays) and made visual observation and identification easier by providing color contrast. For example, an emerald colored reaction plate was well suited for our glucose assay, which used a red indicator. Note, these hydrophobic reaction plates allowed liquid to move freely on the surface. Such plates are a highly desirable alternative to conventional assay / diagnostic platforms because of their cost, ease of use, disposability, and background colors that are helpful to improve detection limits of colorimetric assays. 


\subsection{CALLIGRAPHY PAPER-BASED ANALYTICAL DEVICES}

\subsubsection{Designs of CADs}

A schematic diagram for CAD fabrication process is provided in Figure 1. To establish hydrophobic boundaries, we used a commercial wax printer-a Xerox Phaser $8560 \mathrm{~N}$ color printer-to define assay plate designs in the form of a microzone plate or a hydrophobic reaction plate. The process of wax printing was completed in 5 seconds. After the wax was printed on one side of the paper, we used an oven $\left(105^{\circ} \mathrm{C}\right.$ for $\left.5 \mathrm{~min}\right)$ to melt the wax and allow it to diffuse into the paper and "down" to the other side of the paper to form completely hydrophobic boundaries through the substrate. The microzone plate and hydrophobic reaction plate layouts created 96-well layouts (the diameter of circle was $0.4 \mathrm{~cm}$ ) and $8 \times 7$ arrays (the area of test zone was $0.8 \mathrm{~cm}^{2}$ ), respectively.

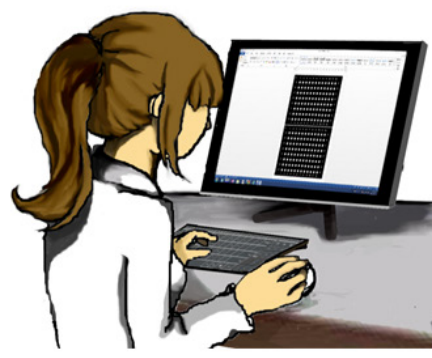

Design pattern
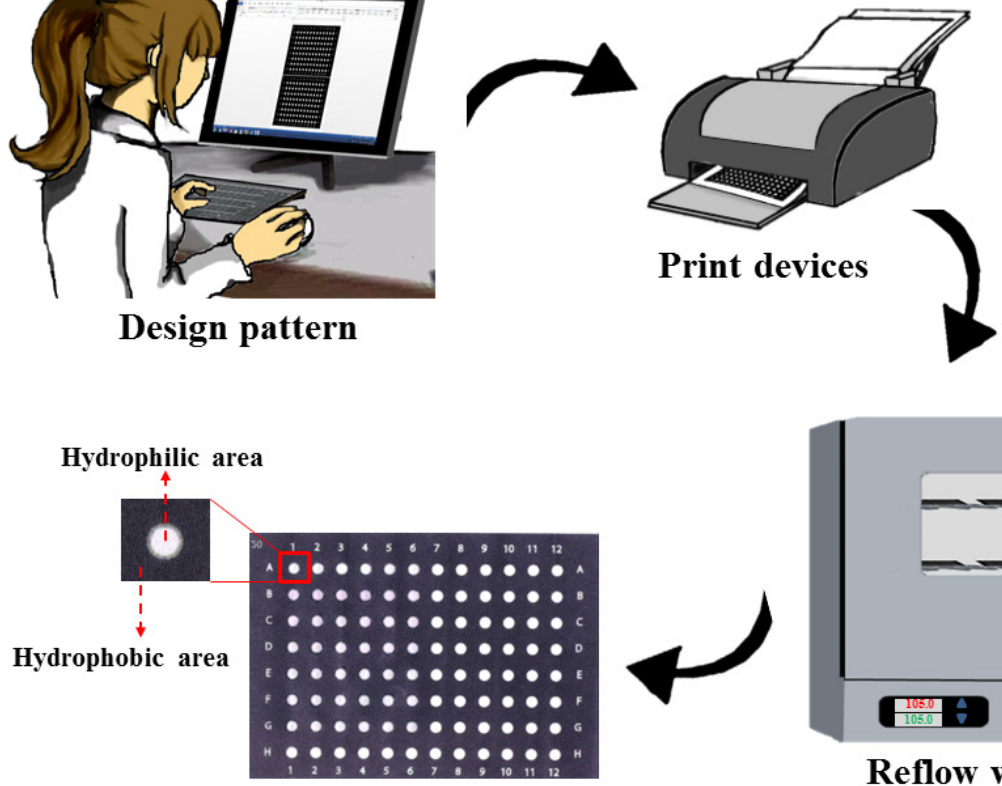

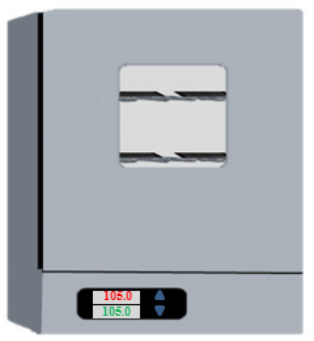

Reflow wax

Figure 1. A schematic diagram of the fabrication process for CADs 


\subsubsection{Physical Properties of CADs}

As for the physical properties of CADs, we have investigated and compared liquid diffusion capabilities for three types of papers (Chinese calligraphy paper, rice paper, and Whatman filter paper No.1) (Figure 2). We pipetted $2 \mu \mathrm{L}$ of red ink solutions (i.e., $0.5 \mathrm{mM}$ and $0.5 \mathrm{M}$ ) onto each well center of Chinese calligraphy paper, rice paper, and Whatman filter paper No.1, respectively, and then continuously observed the diffusion of red ink on the surface of each paper over the course of 0,30 , and $60 \mathrm{~min}$ (note: each experiment repeated twice). At each time point, and for each red ink concentration, we calculated the red ink coverage area for each type of paperwe did not observe paper chromatography in this experiment because the wicking distance within wells was too short for water and red dye separation. The $0.5 \mathrm{mM}$ red ink covered nearly the entire filter paper test zone in less than a minute ( $60 \mathrm{~s}$ ); by contrast, it was difficult to evenly distribute red ink over the test zones of Chinese calligraphy paper and rice paper in a short period of time, but the diffusion of ink on Chinese calligraphy paper was considerably slower than it was for rice paper, as evidenced by examined red dye coverage area [Figure 2(a)]. Nearly $60 \mathrm{~min}$ were required to fill the test zones on Chinese calligraphy paper and rice paper. The coverage ratios (area covered with ink versus area of test zone) over 60 min for the Chinese calligraphy paper were $75 \pm 0.4 \%$ and $89 \pm 1.0 \%$, the coverage ratios for the rice paper were $73 \pm 0.2 \%$ and $70 \pm 0.5 \%$, and the coverage ratios for Whatman filter paper No. 1 were $93 \pm 1.0 \%$ and $88 \pm 0.3 \%$ [mean distribution \pm standard error (S.E).; $N=96$ for each experiment]. For rice paper, the ratio variations were lower than the ratios for filter paper or calligraphy paper, indicating that liquid applied to rice paper was dispersed more uniformly. The coverage rates for Chinese calligraphy paper and rice paper indicate that uniform diffusion following liquid application could be challenging when using a liquid dropping method. To examine the influence of high viscosity, we also applied $0.5 \mathrm{M}$ red ink into well centers of each paper type [Figure 2(b)]. The resulting coverage ratios for Chinese calligraphy paper over $60 \mathrm{~min}$ were $23 \pm 2.2 \%$ and $29 \pm 1.4 \%$, the coverage ratios for the rice paper were $56 \pm 1.1 \%$ and $51 \pm 1.7 \%$, and the coverage ratios for Whatman filter paper No. 1 were $79 \pm 0.5 \%$ and $75 \pm 0.4 \%$ (mean distribution \pm S.E.; $N=96$ for each experiment). The red ink could not fully cover the test zones of Chinese calligraphy paper and rice paper even after $60 \mathrm{~min}$; nevertheless, the rice paper could achieve more than a $50 \%$ coverage ratio while the Chinese calligraphy paper could only achieve a $30 \%$ coverage ratio. According to these results, a small amount of liquid on Chinese calligraphy paper cannot uniformly disperse as it would on filter paper; indeed, much of the applied ink remained in its originally applied position. 
(a)
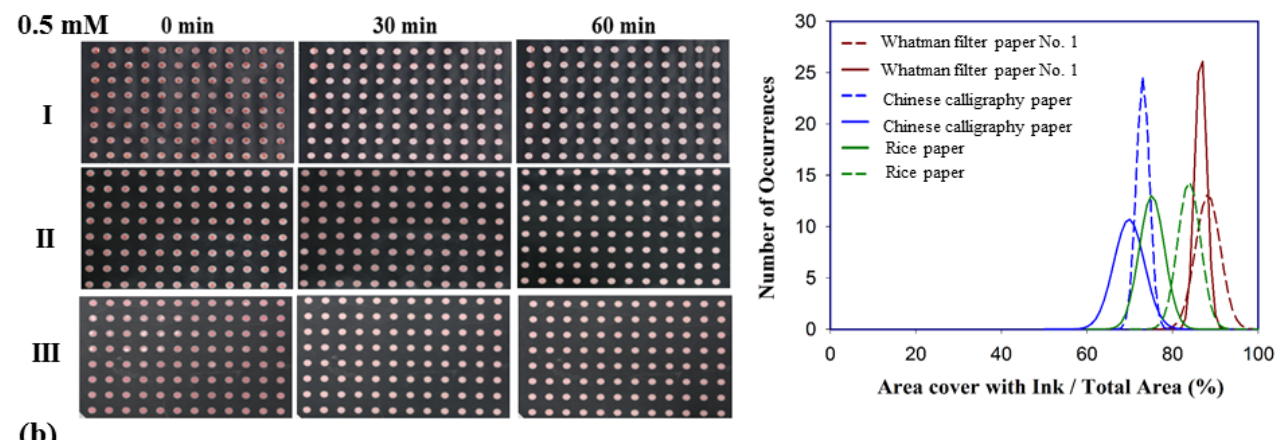

(b)
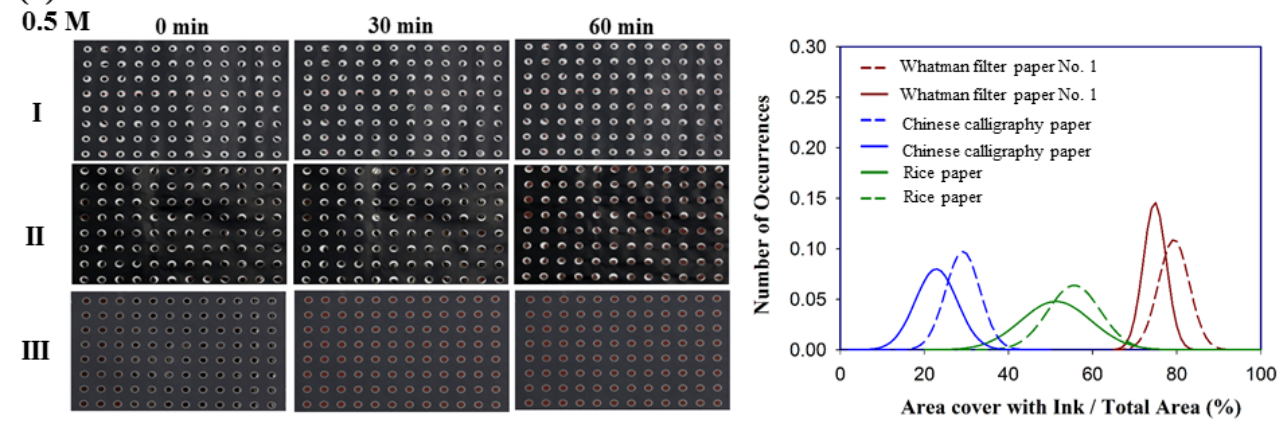

Figure 2. Liquid diffusion test. (a) We added $2 \mu \mathrm{L}$ of $0.5 \mathrm{mM}$ red ink onto the microzone plates that were constructed with calligraphy paper, rice paper, and Whatman filter paper No. 1, respectively, and continuously observed the coverage area change for applied red ink at 0,30 , and $60 \mathrm{~min}$ after spotting. The coverage ratios after 60 min were $75 \pm 0.4 \%$ and $89 \pm 1.0 \%$ for the Chinese calligraphy paper, $73 \pm 0.2 \%$ and $70 \pm 0.5 \%$ for the rice paper, and $93 \pm 1.0 \%$ and $88 \pm 0.3 \%$ for Whatman filter paper No. 1 (mean distribution \pm S.E.; $N=96$ for each experiment). (b) We added $2 \mu \mathrm{L}$ of $0.5 \mathrm{M}$ red ink onto the microzone plates that were constructed of calligraphy paper, rice paper, and Whatman filter paper No 1, respectively, and continuously observed the changes in red ink coverage area at 0,30 , and $60 \mathrm{~min}$ after spotting. After $60 \mathrm{~min}$, the coverage ratios were $23 \pm 2.2 \%$ and $29 \pm 1.4 \%$ for the Chinese calligraphy paper,

$56 \pm 1.1 \%$ and $51 \pm 1.7 \%$ for the rice paper, and $79 \pm 0.5 \%$ and $75 \pm 0.4 \%$ for Whatman filter paper No. 1 (mean distribution \pm S.E.; $N=96$ for each experiment).

To further understand the physical properties of Chinese calligraphy paper, rice paper, and Whatman filter paper No.1, we used a scanning electron microscope (SEM), and energy-dispersive X-ray spectroscopy (EDX) to analyze their topography and elemental compositions [Figure 3(a)]. We discovered that Chinese calligraphy paper and rice paper had more flat surface structures than filter paper in terms of less gaps between and around the surface of fibers. Moreover, we found out that the elemental compositions of Chinese calligraphy paper were divergent from other papers, as it consisted of oxygen, aluminum, silicon, calcium, and palladium atoms instead of the simple 
combination of carbon and oxygen atoms in rice paper and Whatman filter paper No. 1. We propose that the intriguing elemental compositions of Chinese calligraphy paper might be attributable to its unique papermaking process, which takes advantage of a variety of inorganic reagents, such as alum or bleaching agents, to process necessary raw materials [18]. We believe that the distinctive topography and elemental compositions of Chinese calligraphy paper might be crucial to the observed liquid diffusion test differences as compared to rice paper or filter paper.

\subsection{CADs - MICROZONE PLATE}

In addition to physical properties, we used our microzone plate to complete a carbofuran assay in order to investigate the analytical attributes of CADs (note: carbofuran is commonly used as an insecticide, nematicide, and acaricide in agricultural practice [Figure 3(b)] [19].

(a)
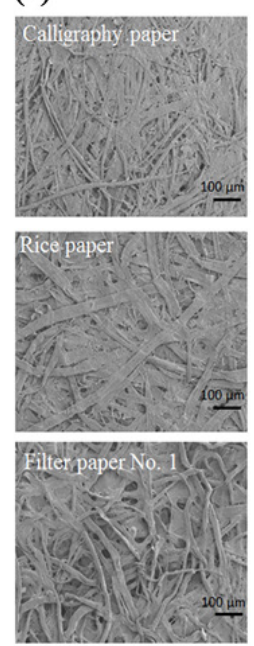
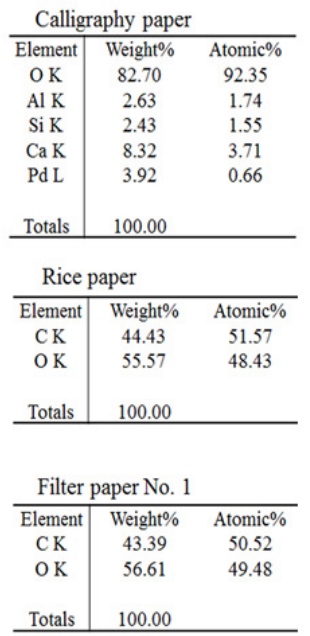

(b)

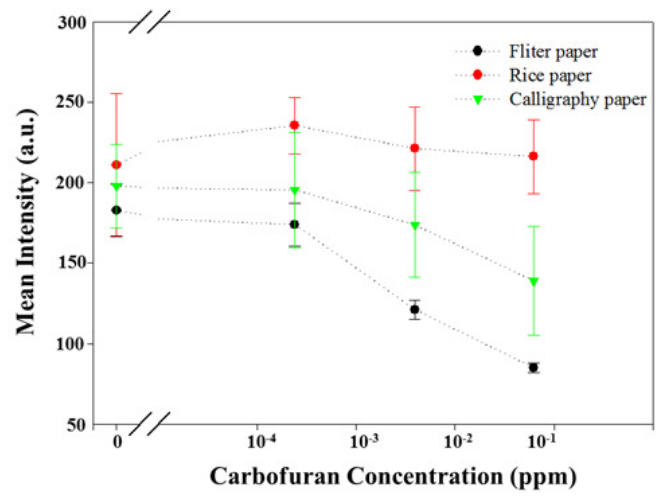

Figure 3. Investigation of the microstructures, elemental compositions, and a carbofuran assay in Chinese calligraphy paper, rice paper, and Whatman filter paper No. 1. (a) Top-view scanning electron microscope images of calligraphy paper, rice paper, and Whatman filter paper No. 1, and the elemental compositions of each paper type from X-ray spectroscopy analysis. (b) The carbofuran assay using microzone plates made of Chinese calligraphy paper, rice paper, and Whatman filter paper No. 1.

The carbofuran assay was conducted using deionized water, and the standard carbofuran solutions for the test were $0,0.0024,0.039$, and $0.625 \mathrm{ppm}$ (Chinese calligraphy paper: $\mathrm{N}=20$; mean intensity \pm S.D., rice paper: $\mathrm{N}=16$; mean intensity \pm S.D., Whatman filter paper No. 1: $\mathrm{N}=16$; mean intensity \pm S.D.). 


\subsubsection{Carbofuran assay}

Carbofuran, an acetylcholinesterase inhibitor, can suppress the enzyme activity of acetylcholinesterase, which catalyzes acetylcholine into choline and acetic acid. The resulting signal, therefore, would degenerate as the levels of carbofuran increased. We added $2 \mu \mathrm{L}$ of carbofuran detection reagent $[1 \mathrm{mM}$ acetylcholine, $3.75 \mathrm{U} / \mathrm{mL}$ acetylcholinesterase, $1 \mathrm{U} / \mathrm{mL}$ choline oxidase, and $0.5 \mathrm{U} / \mathrm{mL}$ hydrogen peroxidase (HRP)] onto each test zone, and waited $5 \mathrm{~min}$ for liquid drying. We subsequently added $2 \mu \mathrm{L}$ of carbofuran standard solutions $(0.0024,0.039$, and $0.625 \mathrm{ppm})$, prepared in deionized water, onto each test zone treated with the detection reagent, and waited $6 \mathrm{~min}$ for the chemical reactions to occur. Finally, we added $2 \mu \mathrm{L}$ of $3,3^{\prime}, 5,5^{\prime}$ tetramethylbenzidine (TMB) solution to colorimetrically indicate the detection results, and we recorded the colorimetric results with a camera (EOS 5D Mark III, Canon, Japan). For result analysis, the recorded images were imported into ImageJ software [a public software from National Institutes of Health (NIH)], which provided the distribution of red, green, and blue colors in our image. We analyzed the color intensity of our carbofuran assay results under the 8-bit setting.

Figure 3(b) shows that the carbofuran assay signal intensity within test zones on rice paper or Chinese calligraphy paper was higher than the signal intensity within test zones on filter paper. Although rice paper had the strongest carbofuran assay signal response, signal changes that should have been obvious across carbofuran concentration variations could not be discerned. There may be two reasons for this: i) the concentration of carbofuran detection reagent in this experiment produced an oversaturated reaction response for each concentration of carbofuran used on our rice paper microzone platecarbofuran assays using Chinese calligraphy paper and filter paper microzone plates employed the same concentration of carbofuran detection reagent; and, ii) the low diffusion capacity of both rice paper allowed the largest amount of carbofuran detection reagent to remain on the applied position rather than spreading throughout the reaction zone, so the detection reaction was confined in a limited space and resulted in an oversaturated response in each experimental setting. While the low diffusion effect also occurred in Chinese calligraphy paper, we believe that the complicated elemental constituents of Chinese calligraphy paper might further hinder some carbofuran assay enzyme reactions. Some research has shown that palladium and aluminum ions may inhibit acetylcholinesterase activity in cells, while calcium ions may enhance it $[20,21]$. Silicon is an inert material for enzymes, which has been introduced into a wide spectrum of sensor applications, so acetylcholinesterase activity would not be influenced by the silicon in Chinese calligraphy paper [22].

Although rice paper and Chinese calligraphy paper microzone plates could produce higher signal intensity than filter paper, large deviations in measurements were likely attributable to non-uniform solution dispersion 
effects and rough manufacturing quality control. We believe that these issues can be improved through fine engineering optimization, i.e., the production of consistent calligraphy paper quality across and among paper batches.

\subsection{CADs - HYDROPHOBIC REACTION PLATE}

For exploring CAD flexibility and utility, we not only examined advantages of the microzone plate, but we studied the capabilities of a completely hydrophobic reaction plate, which was totally covered by wax following wax printing. As a substrate for our hydrophobic reaction plate, we selected Chinese calligraphy paper because it could confine most liquids and produce strong hydrophobic effects if the applied wax generated limited diffusion following wax reflow. In order to confirm the hydrophobic influence of these reaction plates, we made contact angle measurements and conducted a liquid sliding test as proofs (Figure 4). The resulting contact angle measurement for these reaction plates was $107.29 \pm 3.64^{\circ}$ [Figure $\left.4(\mathrm{a})\right] \quad(N=3$; mean angle \pm S.D.). We successfully formed a completely hydrophobic interface on our reaction plate as evidenced by the fact that the contact angle was over $90^{\circ}$ (the standard criteria to determine material hydrophobicity). Figure 4(b) depicts our liquid transport-based nitrite assay. We added a detection reagent $(40 \mu \mathrm{L})$ and a $10 \mathrm{mM}$ nitrite solution $(40 \mu \mathrm{L})$ on the opposite wings of the reaction plate, and then we folded the platform to drive the droplets to move toward the central fold based on the influence of gravity. Afterwards, we could directly observe the color change from colorless to deep red where they uniformly mixed together. Moreover, we added different droplet volumes (i.e., 20,30 , and $40 \mu \mathrm{L}$ ) onto the reaction plate, tilted the device, and observed the droplets smoothly slide from the top part to the bottom part without leaving any solution behind on the surface of the reaction plate. From these results, we determined that the device could be a potential platform for the application of liquid transport studies, such as three-dimensional slippery structures [17] (Figure 5). Although the feasibility of wax coating for MEMs methodology requires further investigation, we did uniquely extend the use of wax printing and wax-printed paper diagnostics by employing hydrophobic background color optimization. 
(a)

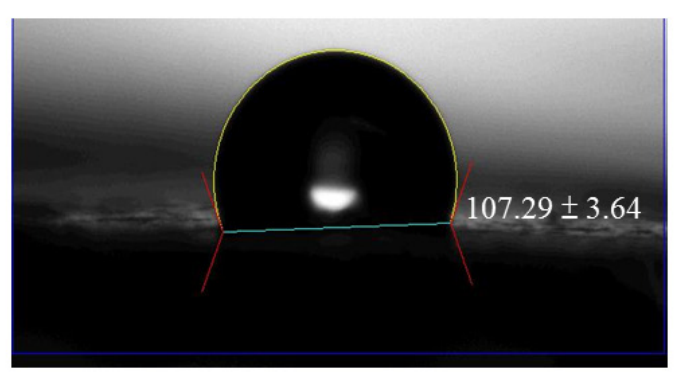

(b)

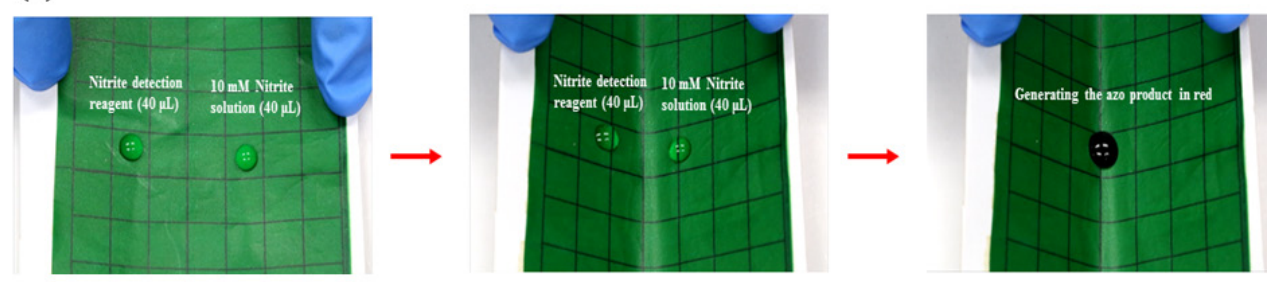

Figure 4. Investigation of the hydrophobicity of calligraphy paper-based analytical devices following wax treatment. (a) The contact angle measurement result was $107.29 \pm 3.64^{\circ}(\mathrm{N}=3$; mean angle \pm S.D. $)$. The surface of calligraphy paper-based analytical devices was shown to be a completely hydrophobic reaction plate after wax treatment, as evidenced by the contact angle measurement, which was greater than

$90^{\circ}$. (b) A nitrite assay demonstration using the idea of liquid transport on the hydrophobic reaction plates. We added detection reagent $(40 \mu \mathrm{L})$ and $10 \mathrm{mM}$ nitrite solution $(40 \mu \mathrm{L})$ on the opposite wings of the device, and folded the device to drive two droplets toward the central part. When they uniformly mixed together, the colorless liquid turned red. 

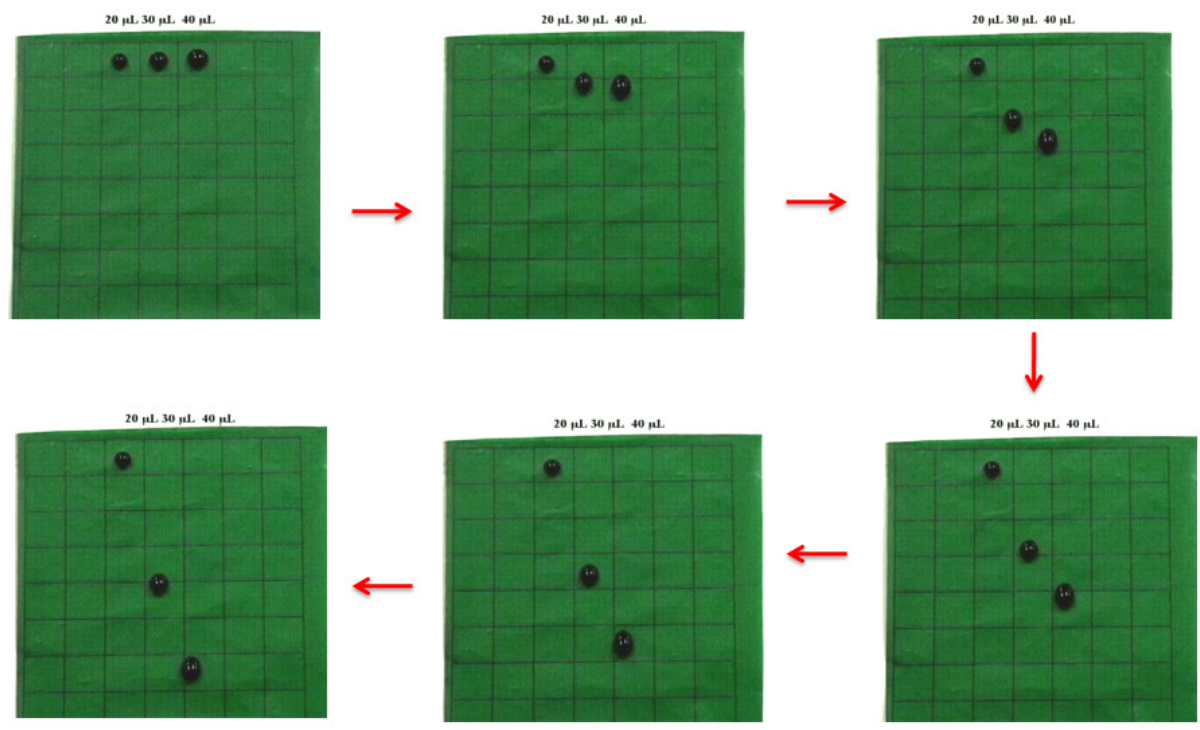

Figure 5. Liquid sliding test. We added 20,30, and $40 \mu \mathrm{L}$ droplets onto the top of our hydrophobic reaction plate and tilted the device. The droplets slid from the top part to the bottom part without leaving any solution behind on the device surface.

(a)

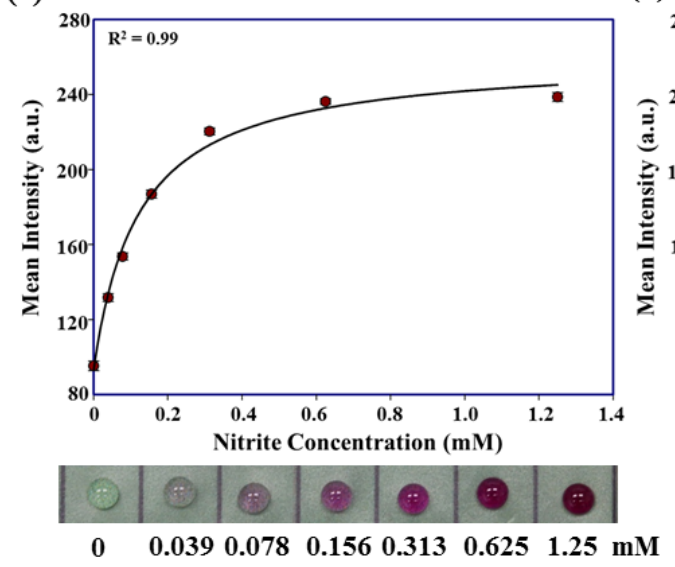

(b)

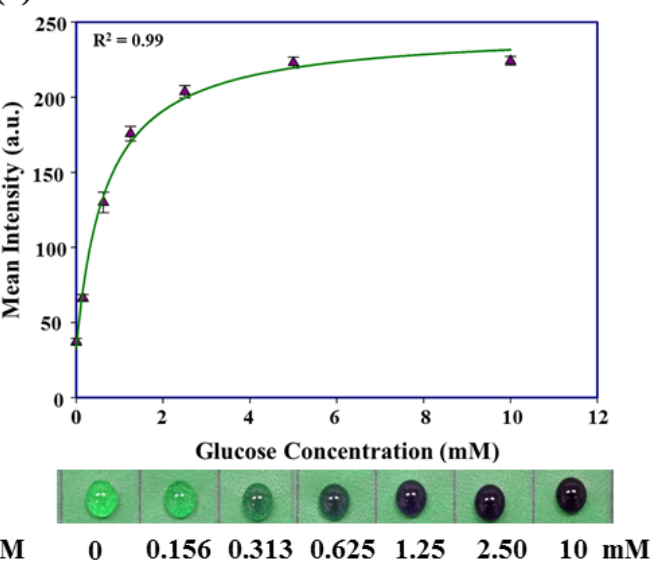

Figure 6. The hydrophobic reaction plates for colorimetric assays. (a) The nitrite assay was conducted using deionized water with $50 \%$ glycerol, and the establishment of a calibration curve under several concentrations-0, 0.039, 0.078, 0.156, 0.013, 0.625, and $1.25 \mathrm{mM}$-resulted. (deionized water: $N=10$; mean intensity \pm S.D.) (b) The glucose assay was conducted using phosphate buffer with $50 \%$ glycerol, and the establishment of a calibration curve under several concentrations- $0,0.156,0.313$, $0.625,1.25,2.50$, and $10 \mathrm{mM}$-resulted. (deionized water: $N=12$; mean intensity \pm S.D.) 


\subsubsection{Nitrite and glucose assays}

We designed our hydrophobic reaction plate based on the idea of providing color contrast for colorimetric assays-the background colors of our reaction plate were designed to contrast with the colorimetric indicators of the assay, which improved readability. Accordingly, we adopted a light grey wax for our nitrite assay, and an emerald wax for our glucose assay (Figure 6). Nitrite and glucose tests are standard items in a regular health examination. In humans, the presence of nitrite ions in urine is a crucial indicator of urinary tract infection severity, and abnormal levels of glucose in blood and urine represent diabetes risk $[23,24]$. The nitrite assay mechanism was based on the Griess reaction. We added $7.5 \mu \mathrm{L}$ of nitrite detection reagent $(25 \mathrm{mM}$ sulfanilamide, $165 \mathrm{mM}$ citric acid, $5 \mathrm{mM} \mathrm{N}$-(1-naphthyl)ethylenediamine dihydrochloride, and $50 \%$ glycerol) onto the test zones of our hydrophobic reaction plate, which was light gray in color, and added $7.5 \mu \mathrm{L}$ of nitrite standard solutions $(0.078,0.156,0.313,0.625,1.25$, and $2.5 \mathrm{mM})$, prepared in deionized water with $50 \%$ glycerol, onto each test zone treated with detection reagent (the final concentrations of nitrite solution for detection were $0.039,0.078,0.156$, $0.313,0.625$, and $1.25 \mathrm{mM}$ due to two-fold dilution via the detection reagent) [Figure 6(a)]. After 3 min of reaction, we recorded the colorimetric results with a camera. In the end, we analyzed the green color intensity for our nitrite assay results.

The glucose assay mechanism was based on the glucose oxidase- and HRPmediated couple reaction. We spotted $7.5 \mu \mathrm{L}$ of glucose detection reagent [300 U / mL glucose oxidase, $60 \mathrm{U} / \mathrm{mL} \mathrm{HRP}, 2 \mathrm{mM}$ 4-aminoantipyrine, $10 \mathrm{mM}$ 4-(Dimethylamino)benzoic acid, $3 \%$ poly(ethylene glycol) (PEG), and $50 \%$ glycerol] onto the test zones of our reaction plate, which was emerald in color, and added $7.5 \mu \mathrm{L}$ of glucose standard solution $(0.313,1.25,2.50,5,10$, and $20 \mathrm{mM}$ ), prepared in deionized water with $50 \%$ glycerol, onto each test zone treated with detection reagent (the final concentrations of nitrite solution for detection were $0.156,0.625,1.25,2.5,5$, and $10 \mathrm{mM}$ due to two-fold dilution via the detection reagent) [Figure 6(b)]. After 4 min reaction, we recorded the colorimetric results with a camera. We then analyzed the green color intensity for our glucose assay results.

Figure 6 demonstrates the results of nitrite and glucose assays from our hydrophobic reaction plates. The LODs for our nitrite and glucose assays were $0.008 \mathrm{mM}$ and $0.08 \mathrm{mM}$, respectively. In healthy people, urine nitrite ions should not be detected in urine samples and urine glucose levels should range from $0-0.8 \mathrm{mM}[23,25]$. The LOD of our nitrite assay was similar to the LOD of PADs $(\mathrm{LOD}=0.005 \mathrm{mM})$ and 5.6 times lower than the LOD of poly(dimethylsiloxane) (PDMS)-based analytical devices (LOD $=0.045 \mathrm{mM}$ ) $[25,26]$. The LOD of our glucose assay was compatible for the current criteria of urine glucose, was better than the LOD of PADs (which could only recognize glucose levels above $0.5 \mathrm{mM}$ ), and was 7 times lower than the LOD of PDMS- 
based analytical devices (LOD $=0.56 \mathrm{mM}$ ) $[27,28]$. We thus believe that integrating color contrast into our platform design significantly improves the LODs of colorimetric assays and readily facilitates identification of results via the naked eye. PDMS is a transparent polymer material manufactured to specific thicknesses for common applications. When it is applied in optical applications, its use is complicated by reflection and deflection, phenomena that increase the signal noise and lower optical analysis. Our platform provides identical functions to that of PDMS-based analytical devices for colorimetrical analysis, i.e., a hydrophobic reaction interface and compatibility with signal recording method via a camera, while eliminating the concern of optical interferences, in turn, advancing the detection limit of a colorimetric assay.

\subsection{CONCLUSION}

We successfully used Chinese calligraphy paper and rice paper in combination with a commercial wax printer to create CADs, including a microzone plate and a hydrophobic reaction plate, for biochemical analysis. Compared to PADs made of Whatman filter paper No.1, we discovered that our microzone plate displayed enhanced carbofuran assay signal (colorimetric assay) due to the low diffusion capacities of both types of papers. However, due to unreliable paper quality, Chinese calligraphy paper and rice paper may cause some difficulties in regards to consistent signal results. Instead of relying on complicated and expensive fabrication, this study used wax printing to explore the ramifications and suitability of hydrophobic treatment on selected paper in terms of handling ease, cost, durability, and sensitivity. The contact angle measurement and liquid sliding test demonstrated that our hydrophobic reaction plate was water resistant and suitable for platforms requiring liquid transport. Notably, we leveraged the idea of color to build a hydrophobic reaction plate with contrasting background colors to the colorimetric indictors used. This greatly improved LODs for our nitrite assay (light gray reaction plate) and our glucose assay (emerald green reaction plate) compared to PADs (5.6 times lower) and PDMS-based analytical devices (7 times lower). 


\section{REFERENCES}

1. A.K. Yetisen, M.S. Akram, C.R. Lowe. Lab chip 13 (2013) 2210-2251.

2. C.-K. Hsu, H.-Y. Huang, W.-R. Chen, W. Nishie, H. Ujiie, K. Natsuga, S.-T. Fan, H.-K. Wang, J.-Y. Lee, W.-L. Tsai, H. Shimizu, C.-M. Cheng. Anal. Chem. 86 (2014) 4605-4610.

3. $\quad$ S.M. Hossain, J.D. Brennan. Anal. Chem. 83 (2011) 8772-8778.

4. H. Wang, Y.-J. Li, J.-F. Wei, J.-R. Xu, Y.-H. Wang, G.-X. Zheng. Anal. Bioanal. Chem. 406 (2014) 2799-2807.

5. N.R. Pollock, J.P. Rolland, S. Kumar, P.D. Beattie, S. Jain, F. Noubary, V.L. Wong, R.A. Pohlmann; U.S. Ryan, G.M. Whitesides. Sci. Transl. Med. 4 (2012) 152 ra129.

6. A.V. Govindarajan, S. Ramachandran, G.D. Vigil, P. Yager, K.F. Böhringer. Lab Chip 12 (2012) 174-181.

7. $\quad$ E. Fu, P. Kauffman, B. Lutz, P. Yager. Sens. Actuators B Chem. 149 (2010) 325-328.

8. E. Carrilho, A.W. Martinez, G.M. Whitesides. Anal. Chem. 81 (2009) 7091-7095.

9. X. Mao, T.-J. Huang, Lab chip 12 (2012) 1412-1416.

10. K. Abe, K. Suzuki, D. Citterio, Anal. Chem. 80 (2008) 6928-6934.

11. F.R. de Souza, G.L. Alves, W.K. Coltro, Anal. Chem. 84 (2012) 9004-9007.

12. M.-Y. Hsu, C.-Y. Yang, W.-H. Hsu, K.-H. Lin, C.-Y. Wang, Y.-C. Shen, Y.-C. Chen, S.-F. Chau, H.Y. Tsai, C.-M. Cheng. Biomaterials 35 (2014) 3729-3735.

13. A. Apilux, Y. Ukita, M. Chikae, O. Chailapakul, Y. Takamura. Lab chip 13 (2013) 126-135.

14. R. Martins, I. Ferreira, E. Fortunato. Phys. Status Solidi RRL 5 (2011) 332.

15. V.L. Pushparaj, M.M. Shaijumon, A. Kumar, S. Murugesan, L. Ci, R. Vajtai, R.J. Linhardt, O. Nalamasu, P.M. Ajayan. Proc. Natl. Acad. Sci. U.S.A. 104 (2007) 13574-13577.

16. J. Kim, S.H. Bae, H.G. Lim, Smart Mater. Struct. 15 (2006) 889.

17. A.C. Glavan, R.V. Martinez, A.B. Subramaniam, H.J. Yoon, R.M.D. Nunes, H. Lange, M.M. Thuo, G.M. Whitesides. Adv. Funct. Mater. 24 (2014) 60-70.

18. G. Chen, K.D. Katsumata, M. Inaba. Restaurator 24 (2008) 135-144.

19. R.C. Gupta. J. Toxicol. Environ. Health 43 (1994) 383-418.

20. J.K. Marquis, G.D. Webb. Mol. Cell. Biochem. 16 (1977) 31-36.

21. T.R. Sharp, T.L. Rosenberry. Biophys. Chem. 21 (1985) 261-264.

22. S. Su, Y. He, M. Zhang, K. Yang, S. Song, X. Zhang, C. Fan, S.-T. Lee. Appl. Phys. Lett. 93 (2008) 023113.

23. S.-C. Lin, M.-Y. Hsu, C.-M. Kuan, H.-K. Wang, C.-L. Chang, F.-G. Tseng, C.-M. Cheng. Sci. Rep. 4 (2014) 6976.

24. American Diabetes Association, Diabetes Forecast (2009). http://www.diabetesforecast.org/2009/apr/checking-your-blood-glucose.html (Nov 14, 2016).

25. U.S. National Library of Medicine, Glucose urine test. http://www.nlm.nih.gov/medlineplus/ency/article/003581.htm (Nov 14, 2016).

26. S.A. Klasner, A.K. Price, K.W. Hoeman, R.S. Wilson, K.J. Bell, C.T. Culbertson. Anal. Bioanal. Chem. 397 (2010) 1821-1829.

27. C.-Y. Yang, C.-M. Kuan, J.A. Yeh, C.-M. Cheng. RSC Adv. 4 (2014) 12538-12544.

28. W. Dungchai, O. Chailapakul, C.S. Henry. Anal. Chim. Acta. 674 (2010) 227-223. 
